\title{
Select papers from the Denver X-ray Conference, additions to the Metal Organic Framework special collection, and exceptional Technical, Educational and Review papers are included in this Issue of PDJ
}

This issue of Powder Diffraction has an unusually broad scope of quality papers that should be of wide interest to PDJ readers. To provide some organization, there are several sections to this issue.

The first section is a set of four papers from contributions to the proceedings of the August 2018 Denver X-ray Conference. They were selected to be highlighted in Powder Diffraction because of their broad interest and range of topics. These include a timely paper titled "3D Immersive Visualization of Micro-Computed Tomography and XRD Texture Data". The other papers are: "Monitoring of $\mathrm{FeS}_{2}$ reactions using High Temperature XRD coupled with Gas Chromatography"; "Implementation of the Self-Consistent Kröner-Eshelby Model for the Calculation of X-ray Elastic Constants for any Crystal Symmetry"; and "INDEXCUB: A Ready-to-Use Set of Routines for X-ray Diffraction Line Profile Analysis". The full proceedings of the conference will be available via the Advances in X-ray Analysis website.

The second section in this issue of Powder Diffraction has two Metal Organic Framework (MOF) related papers. They are the second group of papers and add to those published in the March issue of PDJ. Together they provide a focused Special Section of PDJ that demonstrates the importance of not only powder diffraction but also combining powder diffraction with other characterization methods in characterizing complex materials such as MOFs. The development of new MOFs is very dependent on understanding the crystallographic structure and particularly the channels or tunnels that gas molecules such as $\mathrm{CO}_{2}$ can move through.

The third section of this PDJ issue contains many outstanding papers. I will highlight just three, while recognizing the quality of those not discussed. First, The Crystallography Education Article "Materials for Learning Use of GSAS-II" provides an excellent summary of how to become a user of GSAS-II, which today is a comprehensive tool for nearly all types of structural and material characterization studies. Two other important papers related to the analysis of pharmaceutical materials are included: the Technical Article "Formulation Analyses of High-Volume Prescription Drugs" and the Review Article "A Practical Guide to Pharmaceutical Analyses using X-ray Powder Diffraction". In addition to the three highlighted above, there are three additional Technical Articles covering $\mathrm{XRD}$ analysis of concrete, mineral analysis related to the determination of provenance of Atamandai Type Pottery from 2500 to $1500 \mathrm{BC}$, to structure analysis of a material crystallizing with $Z Z^{\prime}=3$ in the asymmetric unit as well as two outstanding New Diffraction Data papers.

Lastly, during the March meeting of the International Center for Diffraction Data, a PDJ Advisory Board was established with the primary goal of building on the past and making PDJ increasingly valuable to the readers and the wide community of applied material researchers using powder diffraction and related diffraction techniques such as tomography, texture analysis, residual stress analysis, X-ray fluorescence, pair distribution analysis, instrumentation, and software analysis tools. The Advisory Board looks forward to obtaining comments on how to evolve Powder Diffraction so that it is of greater value to our authors and readers. I look forward to receiving your thoughts on this important long-range effort. Please e-mail me at camden.hubbard@me.com. Your input would be greatly appreciated by the Advisory Board and me.

Camden Hubbard Editor-in-Chief, Powder Diffraction camden.hubbard@me.com 